\title{
Lessons Learnt from Managing Orthopaedic Trauma During the First Wave of the COVID-19 Pandemic at a UK District General Hospital
}

\author{
B. A. Patel ${ }^{1,2} \cdot$ S. F. Green ${ }^{1,3} \cdot$ C. Henessy ${ }^{1,4} \cdot$ F. Adamu-Biu ${ }^{1,5} \cdot$ K. Davda $^{6} \cdot$ R. Chennagiri $^{1,6} \cdot$ R. Kankate $^{6} \cdot$ Y. Ghani $^{1,6}$
}

Received: 17 January 2021 / Accepted: 5 May 2021 / Published online: 18 May 2021

(c) Crown 2021

\begin{abstract}
Background The Coronavirus disease (COVID-19) pandemic has contributed to over 1,000,000 deaths worldwide. Hospitals responded by expanding services to accommodate the forecasted rise in COVID-19-related admissions. We describe the effects these changes had on management of orthopaedic trauma and patient outcomes at a district general hospital in Southern England.

Methods Data were extrapolated retrospectively from two separate 6-week periods in 2019 and 2020 (1st April-13th May) using electronic records of patients referred to the orthopaedic team. Soft tissue injuries were included where a confirmed diagnosis was made with radiological evidence. Patients were excluded if no orthopaedic intervention was required. Data were compared between the two time periods.

Results There were fewer attendances to hospital in 2020 compared with 2019 (178 vs. 328), but time from presentation to surgery significantly increased in 2020 (2.94 days vs. 4.91 days, $p=0.009)$. There were fewer operative complications in $2020(36 / 145$ vs. $11 / 88, p<0.001)$. However, ordinal logistic regression analysis found a significantly greater complication severity in 2020 including death $(p=0.039)$. Complication severity was unrelated to COVID-19 status.

Conclusions Restructuring of orthopaedic services in response to the COVID-19 pandemic has been associated with significant delays to surgery and higher post-operative complication severity. Our results demonstrate the need for fast-track emergency operative orthopaedic services in UK district general hospitals whilst the COVID-19 pandemic continues.
\end{abstract}

Keywords COVID-19 - Trauma - Orthopaedics · Demographic data

\author{
B. A. Patel \\ Bhavisha.patel13@nhs.net \\ S. F. Green \\ Sebastian.Green@nhs.net \\ C. Henessy \\ Conor.henessy@nhs.net \\ F. Adamu-Biu \\ Fatima.adamu-biu@nhs.net \\ K. Davda \\ kinner.davda@nhs.net \\ R. Chennagiri \\ rchennagiri@nhs.net \\ R. Kankate \\ raghubir.kankate@nhs.net
}

\author{
Y. Ghani \\ yaser.ghani1@nhs.net \\ 1 Stoke Mandeville Hospital, Buckinghamshire NHS \\ Foundation Trust, Aylesbury, UK \\ 2 Health Education Thames Valley, Oxford School of Surgery, \\ Oxford OX4 2GX, UK \\ 3 Department of Neurology, Southmead Hospital, North \\ Bristol NHS Trust, Bristol BS10 5NB, UK \\ 4 Faculty of Health Sciences, St Catharine's College, Oxford \\ University, Oxford OX1 3UJ, UK \\ 5 John Radcliffe Hospital, Oxford OX3 9DU, UK \\ 6 Department of Trauma and Orthopaedics, Buckinghamshire \\ NHS Healthcare Trust, Amersham HP21 8AL, UK
}




\section{Introduction}

The Coronavirus disease 2019 (COVID-19) global pandemic has infected over 20 million people and contributed to over $1,000,000$ deaths worldwide as of September 18th 2020 [1]. UK hospitals have been required to radically adapt their practice in response to COVID-19 to meet the service demands placed on medical and critical care departments and mitigate virus exposure to patients and staff [2]. For trauma and orthopaedic departments, this response has included redeployment of trainees, conversion to virtual or telephone clinic appointments, routine use of full personal protective equipment (PPE) for operations, and cancellation of non-essential and elective procedures.

Despite such extensive reforms in practice, hospitals have been required to deliver substantive ongoing trauma and orthopaedic emergency care. Although the number of orthopaedic admissions has declined during the COVID-19 pandemic [3], there is a paucity of evidence describing the impacts of hospital reforms on both the management and outcomes of orthopaedic injuries. These data are essential for informing and updating clinical guidelines to optimise care during such an unpredictable and challenging climate.

This article describes how the recent changes made at a busy district general hospital in Southern England in response to the COVID-19 pandemic significantly impacted both management and outcomes of orthopaedic trauma. To our knowledge, this is the first study to describe outcomes of orthopaedic trauma managed during the pandemic. We collected and analysed data from a 6 -week period during the pandemic and compared it with the same time period last year. We describe 6-week outcomes following discharge from hospital and make recommendations for change in the management of orthopaedic injuries during the ongoing pandemic.

\section{Methods}

Data were obtained retrospectively from two separate 6-week periods: 3rd April 2019 to 13th May 2019 and 3rd April 2020 to 13th May 2020. All data were obtained from electronic records and recorded on an anonymised spreadsheet. This project was submitted to the local research and development department at Buckinghamshire NHS Healthcare Trust and approved as a service evaluation. It was deemed that ethical approval was not required for this study.

Confirmed diagnoses of orthopaedic trauma and joint sepsis were included. Soft tissue injuries were included where a confirmed diagnosis was made with radiological evidence, for example muscle and/or tendon ruptures. Patients were excluded if no diagnosis had been made or no orthopaedic input was warranted. Head injuries, chronic vertebral insufficiency fractures, patients referred to tertiary centres and other trusts, and patients followed up at different trusts were excluded. Detection of COVID-19 was confirmed or excluded based on a swab detecting viral RNA by polymerase chain reaction.

The following data were extrapolated from electronic records (Evolve ${ }^{\mathrm{TM}}$, Kainos $\mathrm{Ltd}^{\odot}$ ): total number of cases managed operatively (defined as having a procedure carried out in theatres, even if no incision was made e.g. manipulation under anaesthesia in theatres), injury description by anatomical site, total number of cases managed non-operatively (not requiring transfer to theatre for procedure), total number of cases performed with and without general anaesthesia (GA), mean time from presentation to operation and 6-week complication rates.

For operative cases, complications were classified using a modified version of the Clavien-Dindo-Sink system [4-6] (Table 1). For cases that were managed non-operatively, complication severity was graded from 1 to 5 using an inhouse scoring system based on the Clavien-Dindo-Sink system (Table 2).

Statistical analysis was carried out using GraphPad Prism (GraphPad Software, San Diego, CA) and SPSS (SPSS Inc., Chicago, IL) ${ }^{\mathrm{TM}}$. Chi-squared tests were run to compare proportional outcomes, and an unpaired $t$ test was run to compare time to operation between 2019 and 2020. To investigate whether COVID-19 was associated with a significant change in complication grade, we ran ordinal logistic regression models with year as an independent variable (2019 or 2020) and complication grade [I-V (Clavien-Dindo-Sink) or 1-5 (in-house system)] as a dependent variable. ${ }^{1} \mathrm{We}$ excluded 14 participants from analysis of non-operative complication rates in 2019 because their follow-up was conducted at a separate hospital. Data did not violate assumptions for the statistical methods used.

\section{Results}

There were 328 patients referred to the orthopaedic team in 2019 vs. 178 in 2020. The injury profiles seen in both 2019 and 2020 are described in Fig. 1. Mean sample age was 51.2 years (SD 29.9) for 2019 and 58.2 years (SD 30.1) for 2020. This difference in age was statistically significant $(p=0.006, t=2.76)$. We found that $162 / 328$ referrals (49.4\%) were male in 2019 and 88/178 referrals (49.4\%)

\footnotetext{
${ }^{1}$ Please note that this in-house scoring system is not validated; however, was reviewed and devised by senior orthopaedic clinicians involved in the production of this manuscript.
} 
Table 1 The modified Clavien-Dindo-Sink scoring system for patients undergoing surgery for orthopaedic trauma. Taken from Camino-Willhuber et al. [6]

\section{Grade Definition}

I Non-life-threatening complication that requires transient medication and resolves within the next $72 \mathrm{~h}$

II

Requiring pharmacological intervention for at least $72 \mathrm{~h}$, or active observation without interventions

Unscheduled blood transfusion is also considered

Transient neurological deficit that requires physical therapy with complete recovery

Careful observation of hematoma or seroma

III Requiring endoscopic, radioscopic or surgical treatment

Outside the operating theatre, without or with local anaesthesia (IIIA)

In the operating theatre, with regional or general anaesthesia (IIIB)

IV Life-threatening complication requiring management in the intensive care unit (ICU)

Permanent, unexpected, nerve deficit without recovery

V

Death
Examples of specific complications

Nausea/vomiting/diarrhoea that requires transient medication Superficial phlebitis

Post-operative paralysis resolving completely

Superficial wound infection requiring antibiotics

Transient neuropraxia

Delayed union following femoral or trochanteric osteotomy

Brooker I-II heterotopic ossification; wound complication that does not require treatment

IIIA: prosthetic hip dislocation requiring reduction without anaesthesia

DVT

Knee stiffness not requiring MUA

Abscess that requires percutaneous drainage with/without local anaesthesia

Wound dehiscence that can be treated under local anaesthesia

IIIB: implant loosening

Brooker III-IV heterotopic ossification requiring surgical treatment

Implant failure requiring revision surgery

Periprosthetic fracture including cortical perforations

Joint stiffness requiring MUA

Polyethylene dislocation following total or unicompartmental knee arthroplasty

Avascular necrosis following hip preservation surgery requiring further surgery

Post-operative paralysis from nerve injury without recovery

Compartment syndrome requiring fasciotomy with post-operative Volkmann contracture

Myocardial infarction/pulmonary embolism requiring ICU management

Permanent vascular/neurological injury

Table 2 Our local in-house scoring system for patients undergoing non-operative management for orthopaedic trauma. Based on the modified Clavien-Dindo-Sink classification system for orthopaedic surgery from Camino-Willhuber et al. [6]
Grade Definition
Examples of specific complications

$1 \quad$ Non-life-threatening complication that requires transient medication and resolves within the next $72 \mathrm{~h}$ as a result of hospital admission

2 Requiring pharmacological intervention for at least $72 \mathrm{~h}$, or active observation without interventions

Unscheduled blood transfusion is also considered

Transient neurological deficit that requires physical therapy with complete recovery

3 Requiring endoscopic, radioscopic or surgical treatment

Outside the operating theatre, without or with local anaesthesia (IIIA)

In the operating theatre, with regional or general anaesthesia (IIIB)

4 Life-threatening complication requiring management in the intensive care unit (ICU)

Permanent, unexpected, nerve deficit without recovery

Nausea/vomiting/diarrhoea that requires transient medication Superficial phlebitis

Transient neuropraxia as a result of non-operative management of a fracture

Delayed union following non-operative management

Brooker I-II heterotopic ossification

IIIA: DVT

Knee stiffness not requiring MUA

IIIB: Brooker III-IV heterotopic ossification requiring surgical treatment

Joint stiffness requiring MUA

Paralysis from nerve injury without recovery

Compartment syndrome

Myocardial infarction/pulmonary embolism requiring ICU management

Death 


\section{Orthopaedic injuries by anatomical site and distribution}
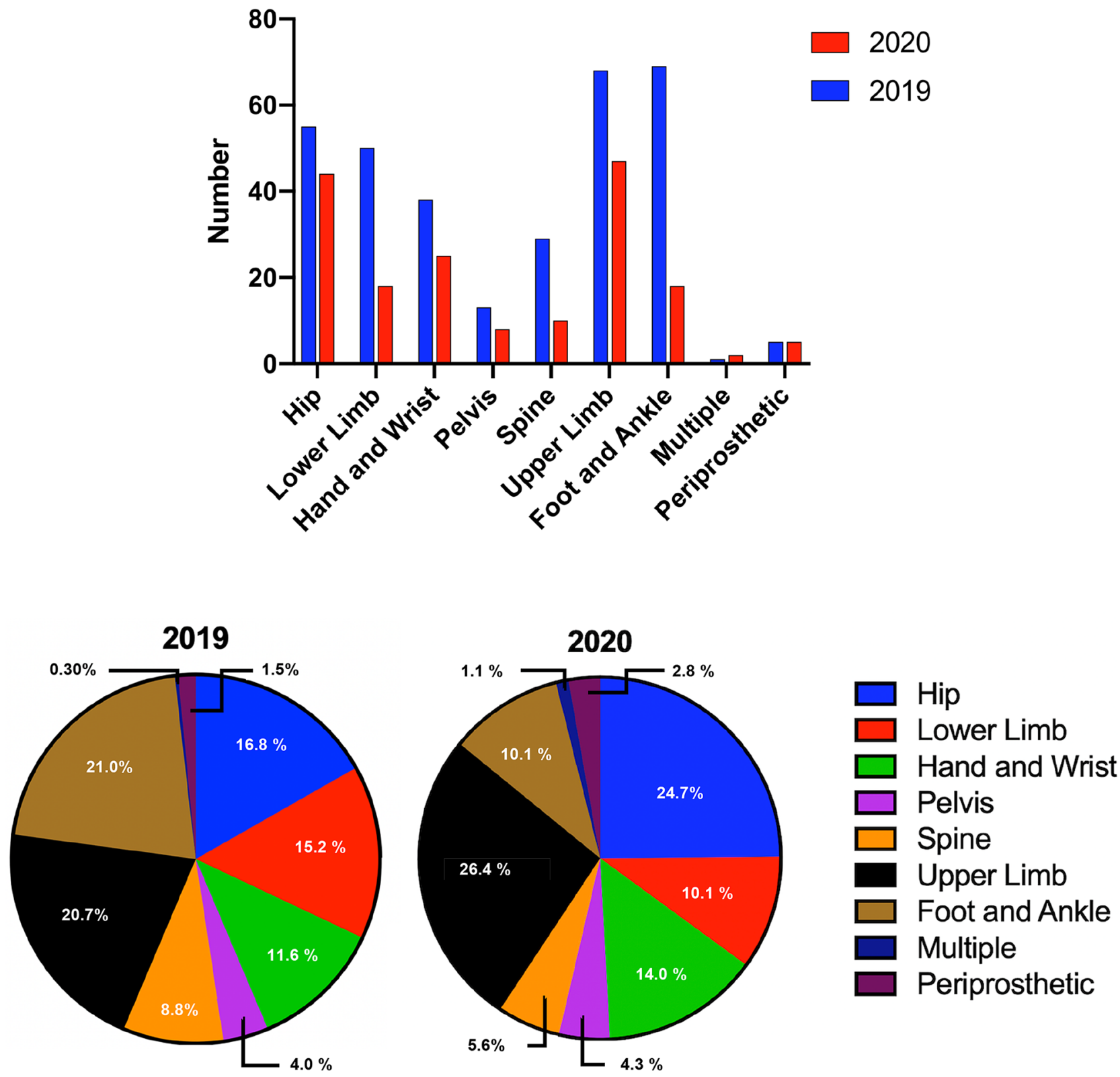

Fig. 1 Orthopaedic injury profile from 6-week periods in 2019 and 2020 and orthopaedic injury profile by distribution from 6-week periods in 2019 and 2020. $n=328$ (2019), $n=178$ (2020)

were male in 2020. There was no significant difference in gender between cohorts $\left(p=0.992, \chi^{2}<0.001\right)$.

There was no significant difference in the proportion of referred cases managed operatively [145/328 (2019) vs. $87 / 178$ (2020); $\chi^{2}=1.258, p=0.262$, Fig. 2]. There was also no significant difference in the proportion of cases managed with general anaesthesia $\left(\chi^{2}=0.305, p=0.581\right)$. A summary of demographic data is displayed in Table 3.

When we looked at whether the COVID-19 pandemic was associated with longer waiting times from presentation to operation, we found a mean waiting time of 4.91 days (SD 4.55) in 2020 and 2.94 days (SD 7.92) in 2019. This difference (1.97 days) was statistically significant $(t=2.401$, $p=0.009$, Fig. 3).

Next, we wanted to test the hypothesis that the COVID19 pandemic was associated with a difference in the overall complication rate of either operative or non-operative management between 2019 and 2020. There were significantly lower complication rates in patients managed operatively in $2020\left[\chi^{2}=5.168, p<0.001 ; 36 / 145(24.8 \% ; 2019)\right.$ vs. $11 / 88$ 


\section{Management of referrals}

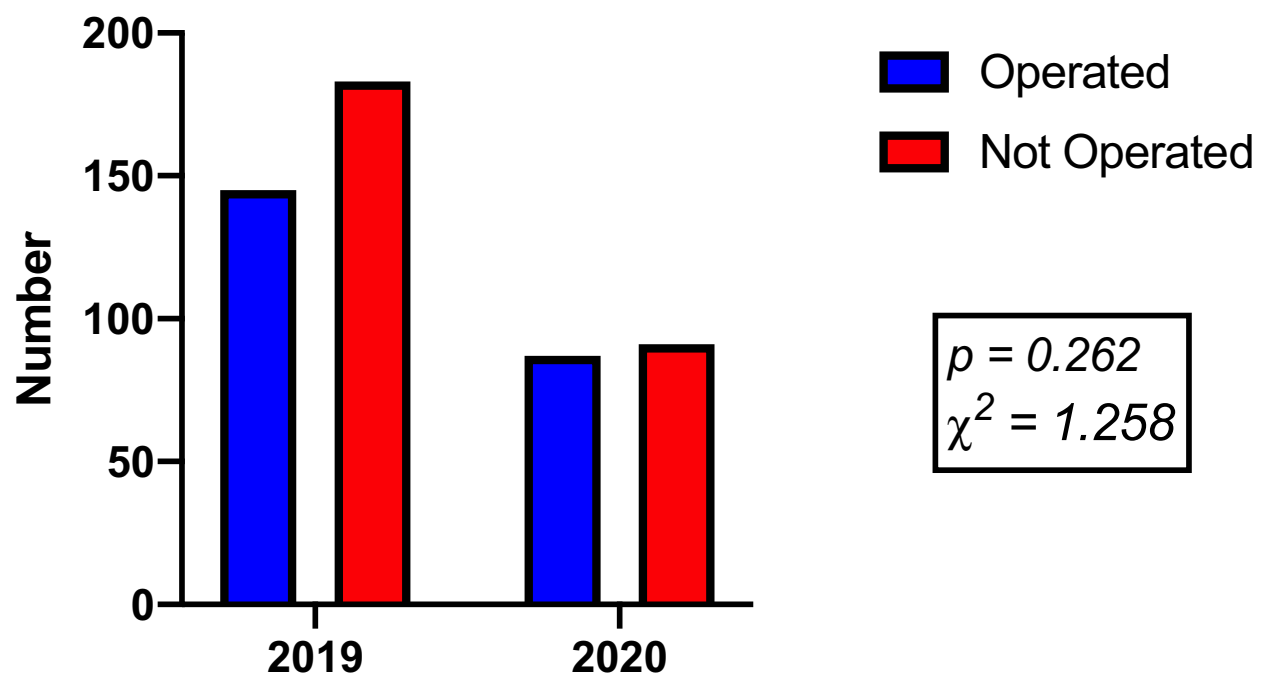

Fig. 2 Management of orthopaedic trauma referrals in 2019 vs. 2020. Referrals from 2019 and 2020 were compared to determine if there was any change in the proportion of cases managed operatively or non-operatively as a result of the COVID-19 pandemic. The results showed that there was no significant change in the proportion of referrals managed operatively in 2019 vs. $2020 * p<0.05, * * p<0.01$
Table 3 Summary of demographic data

\begin{tabular}{llll}
\hline & Year & Statistical significance \\
\cline { 2 - 3 } & 2019 & 2020 & \\
\hline Number of referrals & 328 & 178 & \\
Mean age & 51.2 years (SD 29.9) & 58.2 years (SD 30.1) & $p=0.006, t=2.76$ \\
Gender & 162 male & 88 male & $p=0.992, \chi^{2}<0.001$ \\
Operative management & 166 female & 90 female & \\
General anaesthesia & 145 & 87 & $\chi^{2}=1.258, p=0.262$ \\
\hline
\end{tabular}

The data show there was a significant difference in age between the two cohorts compared in 2019 and 2020

$(12.5 \% ; 2020)]$. This was also the case for non-operatively managed patients $\left[\chi^{2}=3.826, p=0.050 ; 32 / 183(17.4 \%\right.$; $2019)$ vs. $8 / 89(8.99 \% ; 2020)]$.

The next interesting question was whether the COVID-19 pandemic affected the severity of complications suffered following operative or non-operative management. To achieve this, we ran two ordinal logistic regression models with an independent variable of year (2019 or 2020) and an ordinal dependent variable of either the Clavien-Dindo-Sink Classification Grade (I-V), or a classification grade determined in-house for non-operative management (1-5). For post-operative complications, we found a significant overall model fit $\left(\chi^{2}=4.269, p=0.039\right)$. Being operated on during the COVID-19 pandemic significantly increased the odds of having a higher grade of complication on the modified
Clavien-Dindo-Sink scoring system (Odds ratio [95\% CI] 2.100 [1.020-4.327], $p=0.044$; Fig. 4). Since we observed a significant difference in age between 2019 and 2020, we added age as a covariate in this ordinal regression model. Age did not significantly affect the complication severity (OR 0.996 [95\% CI 0.986-1.007], $p=0.499$ ).

When investigating for differences in the severity of complications for patients managed non-operatively, the ordinal logistic regression showed a non-significant overall model fit $\left(\chi^{2}=0.856, p=0.355\right)$. Being managed non-operatively during the COVID-19 pandemic did not significantly change the odds of having a more severe complication (Odds ratio [95\% CI] 0.510 [0.126-2.068], $p=0.346$; Fig. 5).

In 2020, 86/178 (48.3\%) of total referrals had a COVID19 swab test (Table 4). There was no significant difference in 


\section{Time to operation}

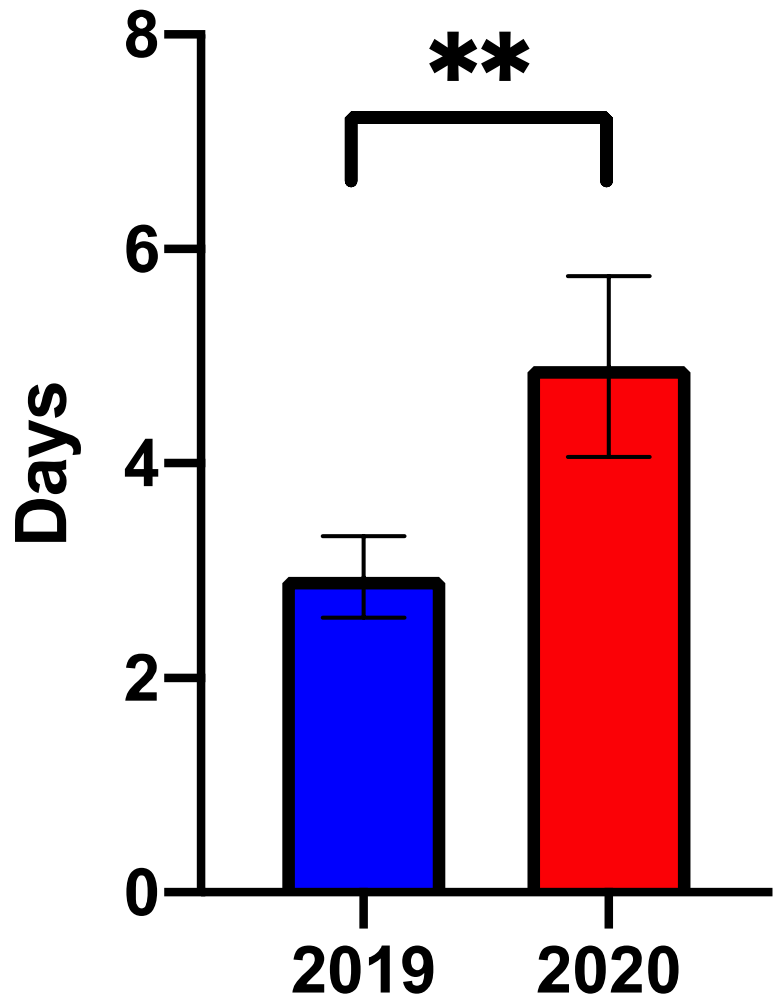

Fig. 3 Time from presentation to the emergency department to operation in 2019 vs. 2020 The data show that during the COVID-19 pandemic, there was a significant increase in the time to operation with a mean wait of 4.91 days in 2020 compared to 2.94 days in 2019 . ${ }^{*} p<0.05, * * p<0.01$ complication severity between COVID-19 positive and negative patients who were managed operatively (Mann-Whitney $U=9.5, p=0.360$ ) or non-operatively (Mann-Whitney $U=1, p=0.400$ ). Out of all six COVID-19-positive patients, one died after developing pneumonitis following a non-operatively managed acetabular fracture on a background of hypertension, ischaemic heart disease, chronic kidney disease and transient ischaemic attack. In 2019, there were a total of seven recorded deaths (five non-operative, two operative). This was also the case for 2020 (three nonoperative and four operative).

\section{Discussion}

This study sought to determine the effects of COVID-19 on the management and outcomes of orthopaedic trauma at a district general hospital in the UK. We found that during the COVID-19 pandemic, there were significantly fewer referrals for orthopaedic trauma. However, patients presenting during the pandemic were significantly older, experienced a significant delay to operation time, and had significantly higher post-operative complication severity including death.

Our finding of a reduction in presentations to the emergency department during the COVID-19 pandemic is consistent with both national and international trends [7-10]. This directly influences the number of orthopaedic injuries cases being managed (either operatively or non-operatively) during the pandemic. The reduction in orthopaedic injury referrals has several possible explanations. First, it may reflect travel restrictions and social curfews resulting in

\section{Operative Complication Grades}

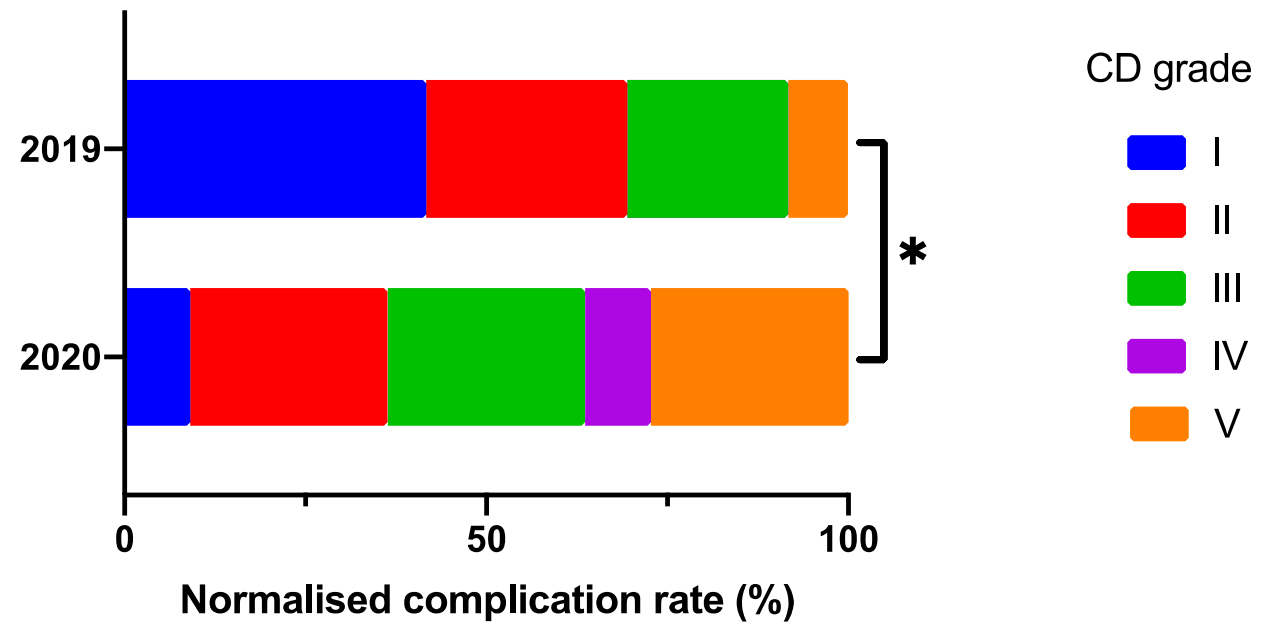

Fig. 4 The spread of complication severity following operative management of orthopaedic trauma during the COVID-19 pandemic. Results are normalised to the overall number of complications $[n=36$ (2019), $n=11$ (2020)]. The data show that during the COVID-19 pandemic, there was a greater occurrence of complications with a higher Clavien-Dindo-Sink grade than in the same period in 2019. $* p<0.05$. $C D$ Clavien-Dindo-Sink 


\section{Non-Operative Complication Grade}

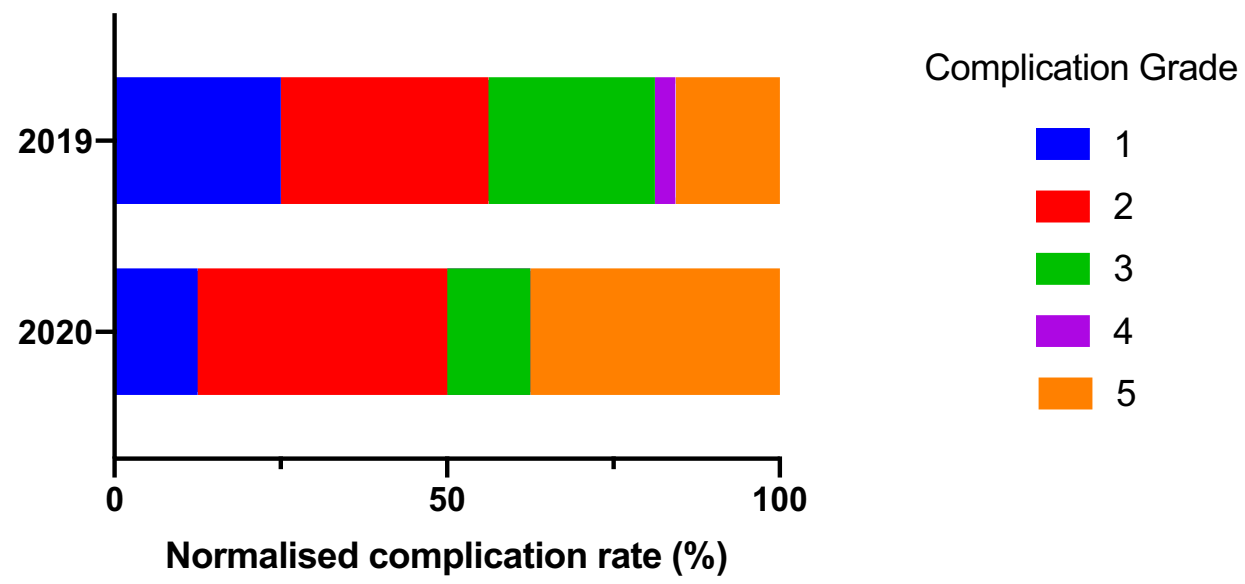

Fig. 5 The spread of complication severity following non-operative management of orthopaedic trauma during the COVID-19 pandemic. Results are normalised to the overall number of complications $[n=32$

Table 4 Results of COVID-19 swabs during 2020

\begin{tabular}{llllr}
\hline & Positive & Negative & Not swabbed & Total \\
\hline Operative & 2 & 54 & 31 & 87 \\
Non-operative & 4 & 26 & 61 & 91 \\
Total & 6 & 80 & 92 & 178 \\
\hline
\end{tabular}

There was no significant relationship between COVID-19 swab results and post-operative outcomes

decreased exposure to physical injury [7]. Second, it may reflect a reduction in work-related injuries associated with more people working from home [7]. Third, it may reflect a reduction in face-to-face primary care consultations leading to fewer referrals from general practitioners [11].

Interestingly, although there was a significantly lower overall complication rate during the pandemic, we found that individuals who were operatively managed in $2020 \mathrm{had}$ significantly increased odds of developing a more severe complication. Concerningly, this includes a higher likelihood of experiencing worse post-operative complications, including death. A possibility is that the severity of injuries and the patterns of trauma observed during the pandemic were different compared to this historical norm. This has been observed by other studies: despite a lower presentation rate to hospital, injuries were more complex in nature compared to the pre-COVID-19 era [12, 13]. We did not find a significant effect of age on complication grade, although there may have been unmeasured differences in comorbidity and case severity between patients operated on in 2019 and 2020, which explains the variance in complication severity. Since there were no individuals who tested positive for
(2019), $n=8$ (2020)]. The data show that during the COVID-19 pandemic, being managed non-operatively was not associated with an increased risk of complication when compared to 2019

COVID-19 at the time of operative management, majority of the differences in complication severity do not appear related to contracting COVID-19. Two patients contracted COVID19 in the post-operative period but remained asymptomatic. This is supported by our observation that having COVID19 did not significantly affect non-operative or operative complication rates. This increased incidence could also be related to high risk and/or essential cases being operated upon during the pandemic. Alternative explanations could be explained by staff shortages owing to contracting COVID-19. As such, the need for expediting trauma patient care whilst ensuring patient and staff safety is paramount in such unprecedented times [14].

Alternatively, the significant increase in post-operative complication severity in 2020 may reflect delays in time to operation. We observed that the pandemic was associated with nearly double the waiting time to operation. Delays in operative management have previously been associated with higher 30-day complication rates and severity following hip fracture [15]. In our study, hip trauma (in particular neck of femur fracture patients) constituted a sizeable proportion of our workload (see Fig. 1). This was also observed in at least one other centre [16]. It is known that delays in operating on this cohort of patients correlate with significantly worse post-operative outcomes [17]. Wignall et al. published a case series at the beginning of the pandemic and highlighted that mortality can be as high as $41 \%$ in this cohort after having contracted COVID-19 [16, 18]. This further highlights the importance of delivering care safely and promptly to these patients and has also been reiterated in at least one metaanalysis [19]. By contrast, other studies have reported no 
difference in functional outcomes for patients with hip fractures who experienced a delay in time to operation [20,21].

An increase in waiting time from presentation to operation during the pandemic has been reported by other orthopaedic and trauma departments $[14,18]$. The delay may reflect hospital policy to wait for a COVID-19 result before operating. Alternative explanations for prolonged waiting times include staff redeployment or the use of full PPE, which results in slower operation turnover [22-24].

This study has some limitations. We did not test all patients for COVID-19, therefore, we cannot fully determine how contracting COVID-19 affected complication grade or severity. During the initial phase of lockdown, not every patient received a COVID-19 swab test. In our operative cohort, approximately $35 \%$ of patients did not have a swab and overall, $52 \%$ of patients were not tested, thereby masking the true effect of contracting the virus. Second, our follow-up is limited to 6 weeks and we, therefore, do not describe long-term patient outcomes and functional status. This is also relevant to the Clavien-Dindo Scoring system that we have used, where complications such as avascular necrosis, knee stiffness, heterotopic ossification and postoperative paralysis may not develop or recover in 6 weeks' duration. Third, we did not control for differences in the complexity or severity of injury and/or surgery observed in 2019 compared with 2020. Lastly, we did not account for the pre-injury status of patients between these two periods. The factors described above may also have contributed to an increase in operative delays.

\section{Conclusion}

The COVID-19 pandemic has resulted in widespread changes to the management and outcomes of orthopaedic injuries at our district general hospital orthopaedic department. Whilst we observed significantly fewer orthopaedic admissions and a lower overall complication rate, we were referred significantly older patients who experienced significant delays to operation associated with significantly increased odds of experiencing more severe post-operative complications. Despite the limitations of our study, our work demonstrates the need for fast-track emergency surgical services during the pandemic to decrease time to operation and reduce post-operative complication severity. This has been described by other orthopaedic departments globally [25, 26].

With the third lockdown in the United Kingdom currently in full swing, the observations and outcomes from this study will encourage departments to manage these orthopaedic trauma cases more efficiently. We recommend earlier testing and faster laboratory processing of COVID-19 swabs to expedite patient care. In addition, full PPE could be reserved for individuals who have a confirmed positive test to minimise delays to operating.

Data Availability If you wish to access the raw data, please contact the corresponding author.

\section{Declarations}

Conflict of Interest The authors declare that they have no conflict of interest.

Ethical Standard Statement This article does not contain any studies with human or animal subjects performed by the any of the authors.

Informed Consent For this type of study, informed consent is not required.

\section{References}

1. Gostin, L. O., Friedman, E. A., \& Wetter, S. A. (2020). Responding to COVID-19: How to navigate a public health emergency legally and ethically keywords. Hastings Center Report, 50(2), 8-12. https://doi.org/10.1002/hast.1090

2. Faria, G., Onubogu, I. K., Tadros, B. J., \& Relwani, J. (2020). Change in practice due to COVID-19-early experiences of a United Kingdom district general hospital in trauma \& orthopaedics. Journal of Orthopaedics, 22, 288-290. https://doi.org/10. 1016/j.jor.2020.06.004

3. Scott, C. E. H., Holland, G., Powell-Bowns, M. F. R., Brennan, C. M., Gillespie, M., Mackenzie, S. P., Clement, N. D., Amin, A. K., White, T. O., \& Duckworth, A. D. (2020). Population mobility and adult orthopaedic trauma services during the COVID-19 pandemic: Fragility fracture provision remains a priority. Bone \& Joint Open, 1, 182-189. https://doi.org/10.1302/2046-3758.16. bjo-2020-0043.r1

4. Iorio, R., Della Valle, C. J., Healy, W. L., Berend, K. R., Cushner, F. D., Dalury, D. F., \& Lonner, J. H. (2014). Stratification of standardized TKA complications and adverse events: A brief communication. Clinical Orthopaedics and Related Research, 472, 194-205. https://doi.org/10.1007/s11999-013-2980-0

5. Sink, E. L., Leunig, M., Zaltz, I., Gilbert, J. C., Clohisy, J., \& Academic Network for Conservational Hip Outcomes Research G. (2012). Reliability of a complication classification system for orthopaedic surgery. Clinical Orthopaedics and Related Research, 470, 2220-2226. https://doi.org/10.1007/s11999-012-2343-2

6. Camino-Willhuber, G., Slullitel, P., Zamboni, D., Albergo, J., Terrasa, S., Piuzzi, N., \& Boretto, J. (2020). Validation of a modified Clavien-Dindo classification for postoperative complications in orthopedic Surgery. Revista de la Universidad Nacional de Cordoba, 77(3), 161-163. https://doi.org/10.31053/1853.0605.v77. n3.27931

7. Leow, S. H., Dean, W., MacDonald-Nethercott, M., MacDonaldNethercott, E., \& Boyle, A. A. (2020). The attend study: A retrospective observational study of emergency department attendances during the early stages of the COVID-19 pandemic. Cureus. https://doi.org/10.7759/cureus.93288

8. Chowdhury, D. (2020). The effects of the COVID-19 pandemic in the UK - at a local, national and international level perspective from the emergency department. Advanced Journal of Emergency Medicine, 4, 4. https://doi.org/10.22114/ajem.v0i0.398 
9. Nunez, J. H., Sallent, A., Lakhani, K., Guerra-Farfan, E., Vidal, N., Ekhtiari, S., \& Minguell, J. (2020). Impact of the COVID-19 pandemic on an emergency traumatology service: Experience at a tertiary trauma centre in Spain. Injury, 51, 1414-1418. https:// doi.org/10.1016/j.injury.2020.05.016

10. Tschaikowsky, T., von Rose, A. B., Consalvo, S., Pfluger, P., Barthel, P., Spinner, C. D., Knier, B., Kanz, K. G., \& Dommasch, M. (2020). Numbers of emergency room patients during the COVID19 pandemic. Notfall \& Rettungsmedizin. https://doi.org/10.1007/ s10049-020-00757-w

11. Duckett, S. (2020). What should primary care look like after the COVID-19 pandemic? Australian Journal of Primary Health, 26, 207-211. https://doi.org/10.1071/py20095

12. Staunton, P., Gibbons, J. P., Keogh, P., Curtin, P., Cashman, J. P., \& O'Byrne, J. M. (2020). Regional trauma patterns during the COVID-19 pandemic. The Surgeon. https://doi.org/10.1016/j. surge.2020.08.003

13. Dhillon, M. S., Kumar, D., Saini, U. C., et al. (2020). Changing pattern of orthopaedic trauma admissions during COVID-19 pandemic: Experience at a tertiary trauma centre in India. Indian Journal of Orthopaedics, 54, 374-379. https://doi.org/10.1007/ s43465-020-00241-0

14. D’Angelo, F., Monestier, L., De Falco, G., et al. (2020). Management of traumatology patients during the coronavirus (COVID19) pandemic: Experience in a Hub Trauma Hospital in Northern Italy. Indian Journal of Orthopaedics, 54, 397-402. https://doi. org/10.1007/s43465-020-00282-5

15. Pincus, D., Ravi, B., Wasserstein, D., Huang, A., Paterson, J. M., Nathens, A. B., Kreder, H. J., Jenkinson, R. J., \& Wodchis, W. P. (2017). Association between wait time and 30-day mortality in adults undergoing hip fracture surgery. JAMA, 318, 1994-2003. https://doi.org/10.1001/jama.2017.17606

16. Samaila, E. M., Negri, S., Corzani, M., Zancan, F., Maluta, T., \& Magnan, B. (2020). The burden of proximal femur fractures and other skeletal injuries during the Covid-19 pandemic lockdown: A retrospective comparative study. Acta Biomedica, 91(14-S), e2020001. https://doi.org/10.23750/abm.v91i14-S.10358

17. Muñoz Vives, J. M., Jornet-Gibert, M., Cámara-Cabrera, J., Esteban, P. L., Brunet, L., Delgado-Flores, L., Camacho-Carrasco, P., Torner, P., Marcano-Fernández, F., \& Spanish, H. I. P. C. I. G. (2020). Mortality rates of patients with proximal femoral fracture in a worldwide pandemic: Preliminary results of the Spanish HIPCOVID observational study. The Journal of Bone and Joint Surgery American, 102, e69. https://doi.org/10.2106/JBJS.20.00686

18. De, C., Wignall, A., Giannoudis, V., et al. (2020). Peri-operative outcomes and predictors of mortality in COVID-19 positive patients with hip fractures: A multicentre study in the UK. Indian
Journal of Orthopaedics, 54, 386-396. https://doi.org/10.1007/ s43465-020-00272-7

19. Kumar Jain, V., Lal, H., Kumar Patralekh, M., \& Vaishya, R. (2020). Fracture management during COVID-19 pandemic: A systematic review. Journal of Clinical Orthopaedics and Trauma, 11, S431-S441. https://doi.org/10.1016/j.jcot.2020.06.035

20. Rai, S. K. (2018). Does time of surgery and complication have any correlation in the management of hip fracture in elderly and can early surgery affect the outcome? European Journal of Orthopaedic Surgery \& Traumatology., 28(2), 277-282.

21. Magaziner, J., Hawkes, W., Hebel, J. R., Zimmerman, S. I., Fox, K. M., Dolan, M., Felsenthal, G., \& Kenzora, J. (2000). Recovery from hip fracture in eight areas of function. The Journals of Gerontology: Series A, 55, M498-M507. https://doi.org/10.1093/ gerona/55.9.M498

22. Meng, Y. T., Leng, K. P., Shan, L., Guo, M., Zhou, J. L., Tian, Q. X., \& Hai, Y. (2020). A clinical pathway for pre-operative screening of COVID-19 and its influence on clinical outcome in patients with traumatic fractures. International Orthopaedics, 44, 1549-1555. https://doi.org/10.1007/s00264-020-04645-3

23. Andreata, M., Faraldi, M., Bucci, E., Lombardi, G., \& Zagra, L. (2020). Operating room efficiency and timing during coronavirus disease 2019 outbreak in a referral orthopaedic hospital in Northern Italy. International Orthopaedics. https://doi.org/10. 1007/s00264-020-04772-x

24. Ti, L. K., Ang, L. S., Foong, T. W., \& Ng, B. S. W. (2020). What we do when a COVID-19 patient needs an operation: Operating room preparation and guidance. Canadian Journal of Anaesthesia, 67, 756-758. https://doi.org/10.1007/s12630-020-01617-4

25. Dabas, V., Bhatia, N., Goel, A., et al. (2020). Management of orthopaedic accidental emergencies amidst COVID-19 pandemic: Our experience in preparing to live with corona. Indian Journal of Orthopaedics, 54, 380-385. https://doi.org/10.1007/ s43465-020-00252-x

26. Adiamah, A., Thompson, A., Lewis-Lloyd, C., Dickson, E., Blackburn, L., Moody, N., Gida, S., La Valle, A., Reilly, J. J., Saunders, J., Brooks, A., \& ICON Trauma Study Group. (2021). The ICON Trauma Study: The impact of the COVID-19 lockdown on major trauma workload in the UK. European Journal of Trauma and Emergency Surgery., 9, 1-9. https://doi.org/10.1007/ s00068-020-01593-w

Publisher's Note Springer Nature remains neutral with regard to jurisdictional claims in published maps and institutional affiliations. 